Global Distribution of Abstracts Presented in HFA Before and During COVID-19 According to First Authors' Country

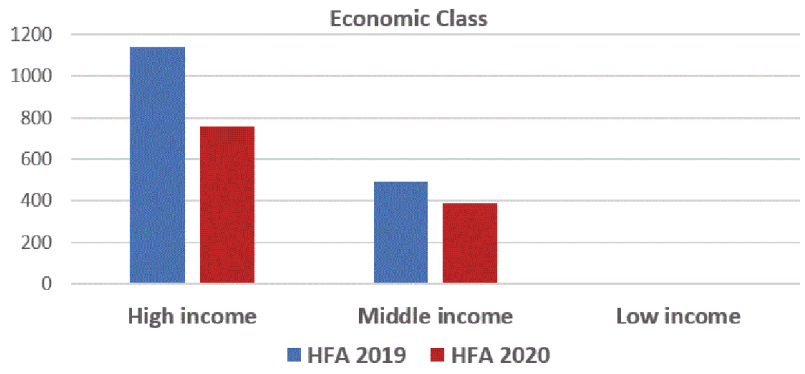

Abstract 149 Figure 2

during COVID-19 era which can be explained by more presentations from these countries due to feasible virtual meetings and waiving of conference fees in 2020 (HFA Discoveries Platform). Further networking and collaborations with researchers from these countries should be encouraged especially with the high cardiovascular disease burden in these countries which necessitated sharing their perspectives and experiences to decrease cardiovascular disease burden worldwide especially by using the hybrid approach and virtual science. Increasing research, travel and educational grants (especially for researchers from LIC) along with calling for action to remove other barriers like VISA issues can help to bridge the conference equity gap and achieve solidarity in science.

Conflict of Interest None

\section{SGLT2 INHIBITION IN HEART FAILURE WITH A REDUCED EJECTION FRACTION: HOW MANY PATIENTS WOULD BENEFIT?}

Jacquelyn Hooper, Steven Shaw, Paul Callan, Simon Williams. Wythenshawe Hospital, Manchester, Manchester, UK

\subsection{6/heartjnl-2021-BCS.147}

Introduction Sodium-glucose co-transporter (SGLT2) inhibition has been shown to reduce the risk of cardiovascular death or hospitalisation in patients with heart failure with reduced ejection fraction (HFrEF) with or without type 2 diabetes mellitus (T2DM). Since publication of the DAPA- $\mathrm{HF}^{1}$ and EMPEROR$\mathrm{HF}^{2}$ trials and UK NICE approval, ${ }^{3}$ SGLT2 inhibitor prescription has been increasing for patients with HFrEF.

Methods Electronic medical records were examined for all patients reviewed in heart failure clinic (including virtual consultation) at Wythenshawe hospital, Manchester over a 4 week period (25/01/21 - 19/02/21). Data obtained was stratified according to NYHA class, LV ejection fraction (LVEF) and presence or absence of T2DM.

Results 232 patients with a diagnosis of heart failure had either face to face or virtual telephone review over the specified time period. Patient baseline characteristics were comparable to patients recruited into the DAPA-HF and EMPEROR-HF trials*. Mean age 67 years (ranging from 20-94 years). $68 \%$ were male. $25 \%$ had T2DM. $19 \%$ $(n=45)$ were subsequently excluded from further analysis due to improvement in LVEF $>40 \%$ (mean time since last echo 5.4 months) or heart failure with preserved ejection fraction, along with 84 patients who were asymptomatic
(NYHA I). 122 patients met the eligibility criteria* for SGLT2 inhibition: of whom 93\% were already established on standard HF treatment (the combination of a betablocker, an aldosternone antagonist or either an ACE inhibitor, or angiotensin II receptor blocker \pm neprolysin inhibitor. $8 \%$ were continuing uptitration of their standard heart failure treatments. In those not on standard HF therapy, a contraindication for the absent therapy was recorded in $50 \%$ of cases. $24 \%(n=29)$ were already prescribed SGLT2 inhibitors. Of the $30 \%(n=37)$ of patients who had T2DM, $43 \%(n=16)$ were prescribed SGLT2 inhibitors, leaving over half without. Amongst the non-diabetic population of eligible patients $(n=86)$, only $15 \%$ were taking SGLT2 inhibitors. Eligible patients taking SGLT2 inhibitors $(n=29)$ were prescribed Dapagliflozin in $79.3 \%$ of cases, with Empagliflozin and Canagliflozin prescribed much less commonly $(10.3 \%$ and $10.3 \%$ respectively). Of those in the SGLT2 cohort, Dapagliflozin was commenced at the most recent clinic appointment in $45 \%$ of cases indicating a rapid uptake in the prescription of these agents.

Conclusion In line with recent NICE guidance, the use of SGLT2 inhibition in the HFrEF population is beginning to increase. There is scope for optimisation in treatment. In our cohort of an unselected group of consecutive patients In heart failure, 122/232 (53\%) patients were eligible for the treatment. Of the patients with T2DM, 57\% were not yet treated with SGLT2 inhibitors. The proportion of nondiabetic eligible patients not treated with SGLT2 inhibition was predictably higher (85\%). However, this figure is likely to fall significantly over the next year as awareness of this new treatment increases and local guidance includes this class of agent.Eligibility criteria* for SGLT2 inhibition included: LV ejection fraction $<40 \%$, symptomatic heart failure (NYHA II or above) and an eGFR $>30$. Those with type 1 diabetes and a history of recurrent UTI were not deemed as eligible.

Conflict of Interest No

\section{REFERENCES}

1. McMurray JJV, Solomon SD, Inzucchi SE, Kober L, Kosiborod MN, Martinez FA Ponikowski P, Sabatine MS, Anand IS, Belohlavek J, et al. Dapagliflozin in patients with heart failure and reduced ejection fraction. $N$ Engl J Med 2019;381:1995-2008. doi: 10.1056/NEJMoa1911303

2. Packer M, Anker SD, Butler J, Filippatos G, Pocock SJ, Carson P, Januzzi J, Verma $\mathrm{S}$, Tsutsui H, Brueckmann M, et al; EMPEROR-Reduced Trial Investigators. Cardiovascular and renal outcomes with empagliflozin in heart failure. $N$ Engl J Med 2020;383:1413-1424. doi: 10.1056/NEJMoa2022190

3. National Institute for Health and Care Excellence NICE technology appraisal guidance TA679.

\section{THE PRE-HFPEF ENTITY: A WINDOW OF OPPORTUNITY TO PREVENT AND HALT THE PROGRESSION TO HF WITH PRESERVED EJECTION FRACTION (HFPEF)}

Patrick Tran, Ross Obukofe, William Thompson, Nimai Desai, Nayaab Abdul Kader, Prithwish Banerjee. University Hospitals Coventry and Warwickshire, Coventry, UK

\subsection{6/heartjnl-2021-BCS.148}

Background Before the emergence of HFpEF, there is an insidious phase of progressive myocardial fibrosis, arterial stiffness, and rising left ventricular end-diastolic pressures (LVEDP) driven by amassing cardio-metabolic comorbidities. This phase is characterized by incipient structural cardiac 
abnormalities but without the manifestation of HF. We hence propose the term 'pre-HFpEF'. (Figure 1) We hypothesize that pre-HFpEF is associated with high morbidity (e.g. hospitalization rate) similar to $\mathrm{HFpEF}$ but without the increased mortality, presuming the ventricle is 'fatigued' but remains compensated. Expanding on preventive cardiology, we believe that early identification of this cohort with aggressive comorbidity control may potentially halt its progression to $\mathrm{HFpEF}$ which has a dismal prognosis and disappointing therapies. Our study therefore aims to demonstrate that this seemingly subclinical entity is clinically diagnosable and depending on the degree of LVEDP, is associated with adverse clinical outcomes.

Methods 1294 consecutive patients with NYHA II-IV breathlessness referred to the community HF clinic were screened and included if LVEF $>45 \%$ and LV diastolic dysfunction (LVDD) were present but without clinical and radiological evidence of HF. Echocardiographic assessment of diastology was based on 2016 EACVI guidelines. 175 patients were

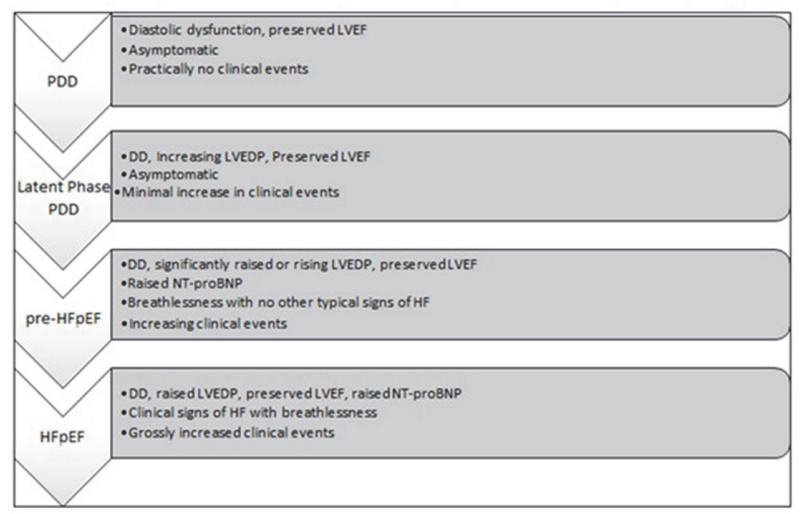

Abstract 151 Figure 1 Proposed spectrum of LV diastolic dysfunction from pie-clinical diastolic dysfunction (PDD) to HFpEF

Abstract 151 Table 1 Comparison of baseline characteristics and clinical outcomes between low and high E/e' groups

\begin{tabular}{|c|c|c|c|}
\hline & $\begin{array}{l}E / e^{\prime}<14 \\
(n=120)\end{array}$ & $\begin{array}{c}E / e^{\prime} \geq 14 \\
(n=55)\end{array}$ & $\begin{array}{c}P \\
\text { value }\end{array}$ \\
\hline \multicolumn{4}{|l|}{ Baseline characteristics } \\
\hline Mean E/e'- (SD) & $9.42( \pm 2.5)$ & $18.77( \pm 6.2)$ & 0.000 \\
\hline Mean NT-proBNP (pmol/L) & 178.47 & 203.37 & 0.022 \\
\hline Hypertension (\%) & $90(75.0)$ & $51(92.7)$ & 0.006 \\
\hline Diabetes (\%) & $30(25.0)$ & $16(29.1)$ & 0.569 \\
\hline Ischaemic Heart Disease (\%) & $31(25.8)$ & $13(23.6)$ & 0.756 \\
\hline Atrial Fibrillation (\%) & $31(25.8)$ & $14(25.5)$ & 0.958 \\
\hline Chronic Kidney Disease (\%) & $17(14.2)$ & $10(18.2)$ & 0.496 \\
\hline Valvular HD (\%) & $40(33.4)$ & $24(43.6)$ & 0.161 \\
\hline Hyperlipidaemia (\%) & $47(39.2)$ & $29(52.7)$ & 0.094 \\
\hline Pulmonary Hypertension (\%) & $23(19.2)$ & $6(10.9)$ & 0.188 \\
\hline Chronic Obstructive Airway Disease (\%) & $23(19.2)$ & $6(10.9)$ & 0.174 \\
\hline Stroke (\%) & $8(6.7)$ & $4(7.3)$ & 0.859 \\
\hline Obstructive Sleep apnoea (\%) & $4(3.4)$ & $5(9.1)$ & 0.103 \\
\hline \multicolumn{4}{|l|}{ Clinical outcomes } \\
\hline $\begin{array}{l}\text { Emergency admissions: } \\
\text { cardiac at } 12 \text { months }\end{array}$ & $22(18.3)$ & $19(34.5)$ & 0.019 \\
\hline $\begin{array}{l}\text { Emergency admission: } \\
\text { non-cardiac at } 12 \text { months }\end{array}$ & $59(49.2)$ & $31(56.4)$ & 0.378 \\
\hline $\begin{array}{l}\text { Length of Stay (days) for cardiac } \\
\text { admissions- median (IQR) }\end{array}$ & $1.7( \pm 5.2)$ & $3.9( \pm 9.6)$ & 0.037 \\
\hline All-cause mortality at 12 months & $10(8.4)$ & $5(9.1)$ & 0.868 \\
\hline All-cause mortality 36 months & $41(34.2)$ & $13(23.6)$ & 0.163 \\
\hline
\end{tabular}

included and divided into two groups: rising LVEDP (E/e' $<14)$ versus raised LVEDP $\left(\mathrm{E} / \mathrm{e}^{\prime} \geq 14\right)$. Clinical demographics and events (deaths, unplanned hospitalization and length of hospital stay) were retrospectively compared at 12 and 36 months.

Results 55 patients had E/e' $\geq 14$ and 120 had E/e' $<14$ with the former group having significantly higher NT-proBNP and proportion of hypertension. There were no significant differences in the other comorbidities usually associated with HFpEF. (Table 1) This may support the concept that pre-HFpEF is at the early stages of disease but has the potential to progress to HFpEF through additional comorbidity burden. The $E / e^{\prime} \geq 14$ group had a significantly higher number of cardiovascular-related admissions and twice the average length of hospital stay compared with the E/e' $<14$ group. At 3 years, the high E/e' cohort had 285 all-cause hospital admissions and 54 all-cause deaths. There was no significant difference in mortality between the two groups at 12 or 36 months, reflecting the notion that pre-HFpEF is at the beginning of the spectrum of both disease and prognosis.

Conclusion A high $\mathrm{E} / \mathrm{e} \geq 14$ cut-off with raised NT-proBNP may be used as a screening tool to identify high-risk preHFpEF group which is associated with adverse clinical outcomes. Without increased mortality so far, it is crucial to promptly identify this population that may be more amenable to risk factor modification than HFpEF. Close monitoring in a dedicated HFpEF clinic with vigorous management of comorbidities is therefore recommended.

Conflict of Interest None

\section{DOES OUTPATIENT BASED IV DIURETIC TREATMENT FOR ACUTE HEART FAILURE GIVE PATIENTS HOPE?}

${ }^{1}$ Kenneth Wong, ${ }^{2}$ D.A. Hughes, ${ }^{3}$ Maciej Debski, ${ }^{3}$ Nang Latt, ${ }^{3}$ Omar Assaf ${ }^{3}$ Amr Abdelrahman, ${ }^{3}$ Rebecca Taylor, ${ }^{4}$ Victoria Allgar, ${ }^{3}$ Loie McNeill, ${ }^{3}$ Stuart Howard, ${ }^{3}$ Suzanne Wong, ${ }^{3}$ Robert Jones, ${ }^{3}$ C.J. Cassidy, ${ }^{3}$ Alison Seed, ${ }^{3}$ Gavin Galasko, ${ }^{5}$ Andrew Clark, ${ }^{6}$ David Wilson, ${ }^{7} \mathrm{G}$ Davis, ${ }^{7}$ Alexander Montasem, ${ }^{8} \mathrm{CC}$ Lang, ${ }^{9}$ Paul Kalra,

${ }^{10}$ R. Campbell, ${ }^{11} \mathrm{G}$. Lip, ${ }^{10}$ John Cleland. ${ }^{1}$ Blackpool Teaching Hospitals, Blackpool, UK; ${ }^{2}$ Centre for Health Economics and Medicines Evaluation, Bangor University, UK; ${ }^{3}$ Blackpool Victoria Hospital; ${ }^{4}$ Professor of Medical Statistics and Director of Peninsula Clinical Trials Unit; ${ }^{5}$ Hull York Medical School; ${ }^{6}$ Worcerstershire Acute Hospital NHS Trust; ${ }^{7}$ School of Medicine, University of Central Lancashire, Preston, UK; ${ }^{8}$ University of Dundee; ${ }^{9}$ Portsmouth Hospitals University NHS Trust; ${ }^{10}$ University of Glasgow, UK; ${ }^{11}$ Liverpool Centre for Cardiovascular Science, University of Liverpool and Liverpool Heart \& Chest

\subsection{6/heartjnl-2021-BCS.149}

Introduction Acute heart failure (AHF) hospitalisation is typically lengthy, costly and associated with $10 \%$ mortality. Outpatient based management (OPM) of AHF appeared effective in observational studies. We conducted a pilot randomised controlled trial (RCT) comparing OPM with standard inpatient care (IPM). Hopelessness independently predicted mortality in patients with risk factors of ischaemic heart disease. We hypothesize that patients randomised to OPM may be more hopeful and have better mental well-being, with better quality of life, patient and carer satisfaction.

Methods We randomised patients with AHF, considered to need IV diuretic treatment for $>2$ days, to IPM or OPM. We recorded all-cause mortality (index-episode), and the number of full days alive and out-of-hospital (DAOH) within 60 days after randomisation. Quality of life (EQ5D-VAS), mental well- 